\title{
Détermination expérimentale du flux d'érosion éolienne
}

\author{
Hugo MARTINEZ, Mohamed NAAIM, Florence NAAIM-BOUVET
}

CEMAGREF (1)

\section{I 물 LE MODE OPÉRATOIRE}

Le dispositif expérimental que nous avons conçu est constitué d'un capteur de distance Keyence LB-60 qui assure la mesure de l'épaisseur du lit de particules (fig l). Ce capteur est composé d'un émetteur (une diode laser d'une longueur d'onde de $780 \mathrm{~nm}$ ) et d'un photo-détecteur. L'image de l'émetteur laser est projetée, par l'intermédiaire d'une optique adéquate, sur le dépôt de particules, et une partie de l'énergie diffusée est focalisée sur le photo-détecteur. Cette diode est connectée à une carte d'acquisition pilotée par le logiciel Asyst sur un PC. Il est donc possible, connaissant le signal fourni par le photo-détecteur, de déterminer avec une précision de $10 \mathrm{~mm}$ l'épaisseur du lit de particules et sa variation dans le temps.

Il est très important de disposer d'un lit de particules d'épaisseur homogène. Pour ce faire on a réalisé un système mécanique qui permet d'atteindre cette exigence pour différentes épaisseurs. Pour s'assurer que, pendant les mesures, les couches de saltation et de suspension ne perturbent pas les mesures, nous avons réalisé plusieurs essais pour lesquels l'épaisseur est mesurée avec et sans transport. Ces essais ont montré que la dispersion de la lumière laser due au transport est négligeable. Les particules $\mathrm{A}, \mathrm{B}$, et $\mathrm{C}$ utilisées dans ces expériences ont des diamètres moyens de 170, .132 et $112 \mu \mathrm{m}$ et des masses volumiques de 544, 591 et $1410 \mathrm{~kg} / \mathrm{m}^{3}$.

\section{EVOLUTION TEMPORELLE DU LIT ET REPRODUCTIBILITÉ DES ESSAIS}

Le flux vertical prélevé par l'écoulement est directement proportionnel à la variation temporelle de l'épaisseur du lit, le coefficient de proportionnalité étant la masse volumique du milieu. Avant toute analyse, nous nous sommes intéressés à la reproductibilité des mesures. Nous avons réalisé différentes mesures dans des conditions expérimentales identiques. Un exemple de ces essais est présenté sur la figure 2, où sont tracées deux courbes représentant l'épaisseur du lit en fonction du temps.

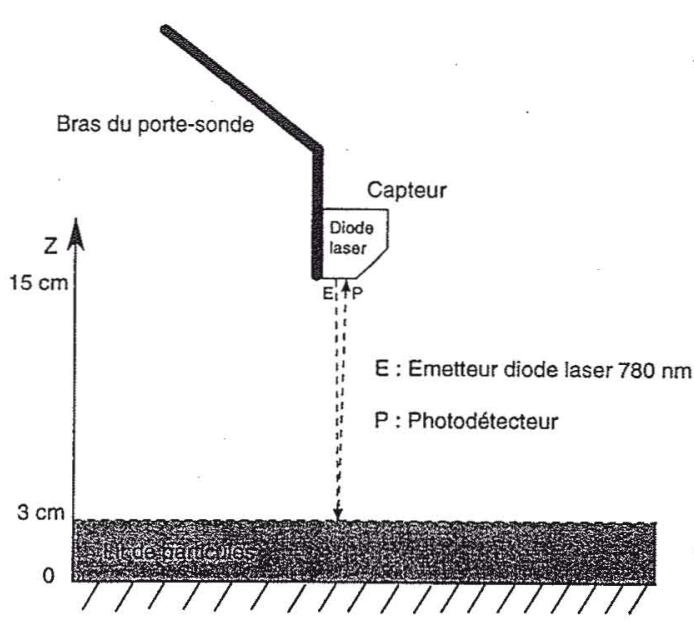

1 - Dispositif de mesure de I'épaisseur du lit de particules.

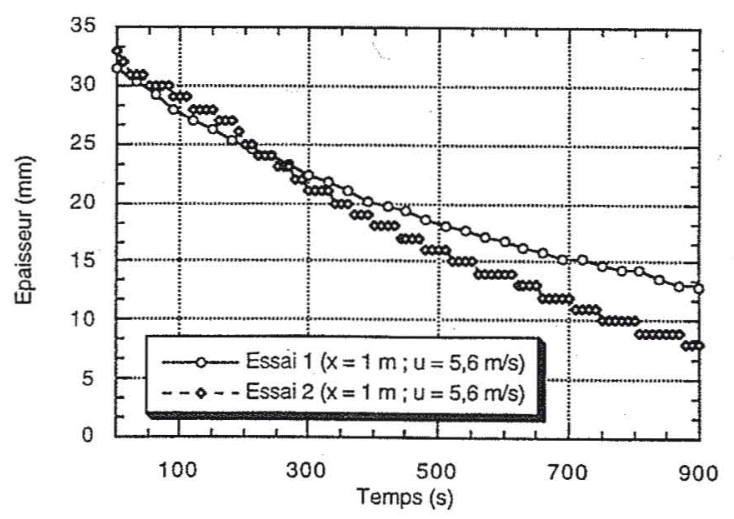

2 - Deux évolutions du lit obtenues dans les mêmes conditions expérimentales.

(1) Division Nivologie - CEMAGREF BP 76 - 38402. Saint Martin d'Hères. France 
A partir des mesures, on peut faire trois observations importantes:

- la présence de rides ou «ripples», formes éoliennes à petite échelle dont la longueur d'onde (ou espacement régulier entre deux formes analogues) est indépendante du temps (fig 2) ;

- la diminution de l'érosion vers "l'aval" de la zone d'étude ; - l'augmentation de l'érosion avec la vitesse de l'écoulement.

Ces trois phénomènes vont être analysés et précisés dans les paragraphes suivants.

\section{E PHÉNOMENE DES RIPPLES}

La surface initiale du lit de particules est toujours horizontale et plane. Les mesures en fonction du temps montrent, pour tous les essais, une évolution plus ou moins rapide vers une surface «ondulée» produite par le phénomène de reptation (les grains sont trop gros pour être emportés par le vent, et leur mouvement est donc induit par les impacts de saltation [1]. Ces ondulations ont des formes semblables aux "ripples". La translation de ces formes éoliennes engendre des ondulations de longueur d'onde, de vitesse de propagation et d'amplitude particulières. Elles ont une longueur d'onde de 5 à $20 \mathrm{~cm}$, leur hauteur ou amplitude varie entre 2 et $5 \mathrm{~cm}$, et elles se déplacent avec une vitesse de migration comprise entre 5 et $20 \mathrm{~cm} / \mathrm{min}$. Si on les observe d'en haut, ces "ripples" ont des crêtes sinueuses. Normalement leur morphologie est très semblable à celle des «ripples» modelées dans le sable [2] (fig 3).

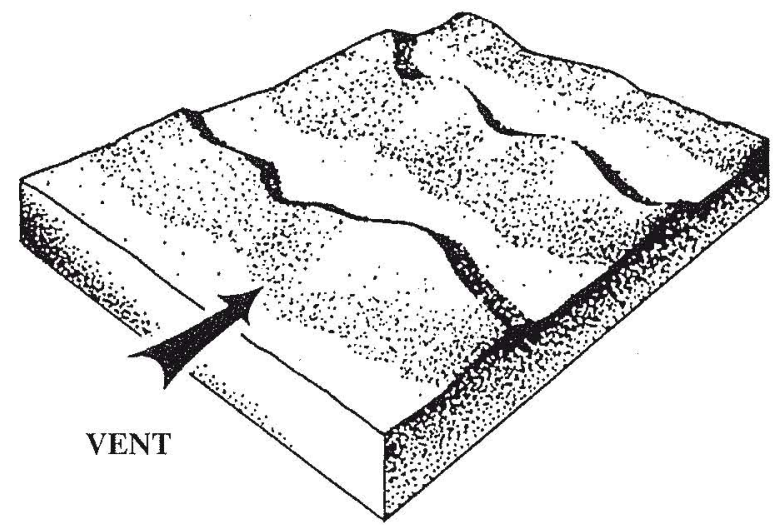

3 - Schéma des rides se formant perpendiculairement à la direction du vent.

\section{1 Evolution spatiale des ripples}

Sur le graphique suivant (fig. 4) sont tracées les évolutions temporelles de l'épaisseur du lit de particules obtenues pour une même vitesse d'écoulement $(5,6 \mathrm{~m} / \mathrm{s})$ aux quatre points de mesure.

A partir de ce graphique on montre que, dès la position $x=2 \mathrm{~m}$, le phénomène apparaît. Ce phénomène a une fréquence plus importante au niveau du deuxième point de mesures. Cette fréquence diminue à la troisième et à la quatrième position. Ceci traduit le caractère transitoire du phénomène qui n'a pas encore atteint un régime établi

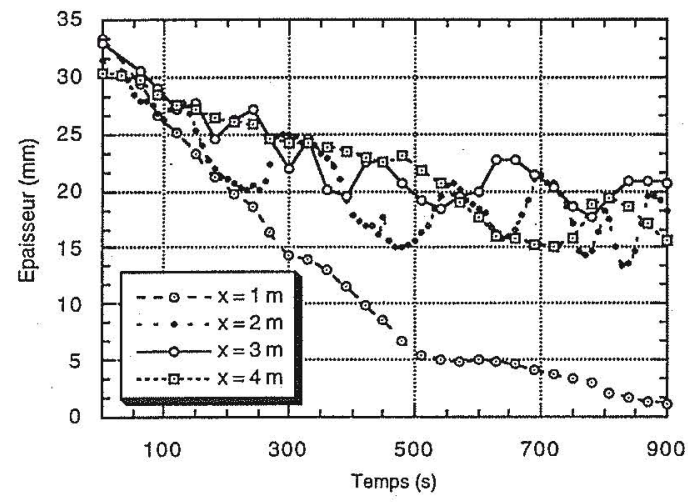

4 - Apparition des «ripples».

\section{2 Rôle de la vitesse de l'écoulement}

Sur le graphique suivant (fig. 5) sont tracées pour le deuxième point de mesure, les évolutions de l'épaisseur du lit de particules pour deux vitesses d'écoulement $(5,6 \mathrm{~m} / \mathrm{s}$ et $8 \mathrm{~m} / \mathrm{s})$.

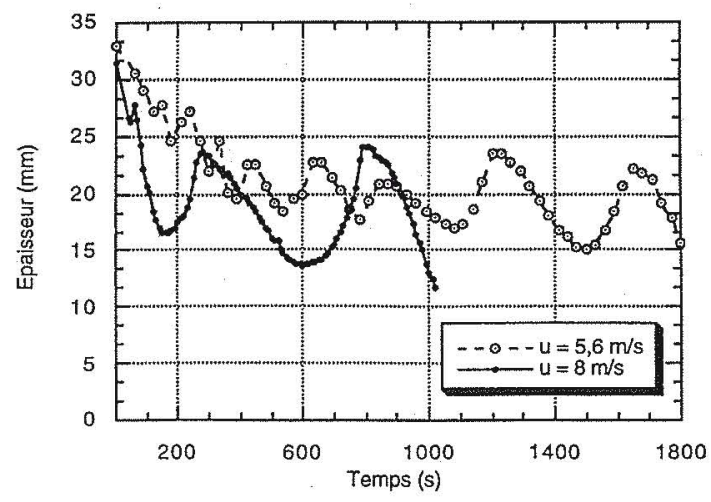

5. Influence de la vitesse d'écoulement sur l'épaisseur du lit de particules.

A faible vitesse, le phénomène met plus de temps pour atteindre une amplitude importante. L'amplitude croît avec la vitesse alors que la fréquence de sa variation temporelle diminue (la longueur d'onde augmente). Ces résultats sont en accord avec les dernières avancées théoriques en matière de formation de ripples présentées par Anderson [3]. En effet, cet auteur a montré que la cause de la formation des ripples est le mouvement par reptation. Lorsque la vitesse du vent augmente, la longueur moyenne de reptation augmente, et par conséquence la longueur d'onde et l'amplitude du ripple aussi. 


\section{FLUX VERTICAL DE PARTICULES DANS LA ZONE NON SATURÉE}

La dérivée temporelle moyenne de l'épaisseur du lit de particules $\left(\mathrm{e}_{\mathrm{p}}\right)$ nous permet de déterminer le flux d'érosion comme :

$$
\phi_{\text {érosion }}(x)=\left.\rho_{\text {app }} \frac{d\left(e_{p}\right)}{d t}\right|_{x}
$$

où $\rho_{\text {tipp }}$ représente la masse volumique apparente due à la porosité du lit $\eta(\eta=60 \%)$. Elle est calculée par :

$$
\rho_{\mathrm{app}}=\rho_{\mathrm{p}}(1-\eta)
$$

Nous avons réalisé différents essais pour différentes caractéristiques des particules. Ces essais ont permis d'analyser différentes évolutions du flux de masse érodée.

\subsection{Evolution spatiale de l'érosion}

Dans le cas des particules $\mathrm{A}$, et pour les vitesses d'écoulement de $5,2 \mathrm{~m} / \mathrm{s}$ et $5,6 \mathrm{~m} / \mathrm{s}$, le tableau 1 et la figure 6 montrent l'évolution du flux de masse érodée par l'écoulement en fonction de la distance dans le sens de l'écoulement.

\begin{tabular}{|c|c|c|c|c|}
\hline Vitesse & $\begin{array}{c}\text { Flux à } \\
\mathrm{x}=1 \mathrm{~m}\end{array}$ & $\begin{array}{c}\text { Flux à } \\
\mathrm{x}=2 \mathrm{~m}\end{array}$ & $\begin{array}{c}\text { Flux à } \\
\mathrm{x}=3 \mathrm{~m}\end{array}$ & $\begin{array}{c}\text { Flux à } \\
\mathrm{x}=4 \mathrm{~m}\end{array}$ \\
\hline $5,2 \mathrm{~m} / \mathrm{s}$ & 0,043 & 0,022 & 0,009 & 0,005 \\
\hline $5,6 \mathrm{~m} / \mathrm{s}$ & 0,054 & 0,029 & 0,016 & 0,007 \\
\hline
\end{tabular}

Tableau 1. - Flux de masse érodée en $\mathrm{kg} / \mathrm{m}^{2} \mathrm{~s}$.

A partir de ce tableau on peut tirer les conclusions suivantes:

- le flux de masse est d'autant plus important que la vitesse d'écoulement est forte ;

- le flux vertical massique diminue quasi-linéairement entre le début et la fin de la veine. A la quatrième position il est à peine égal à $10 \%$ du flux vertical mesuré à $\mathrm{x}=1 \mathrm{~m}$. Ce résultat tend à montrer que la saturation est assez proche (fig 6).

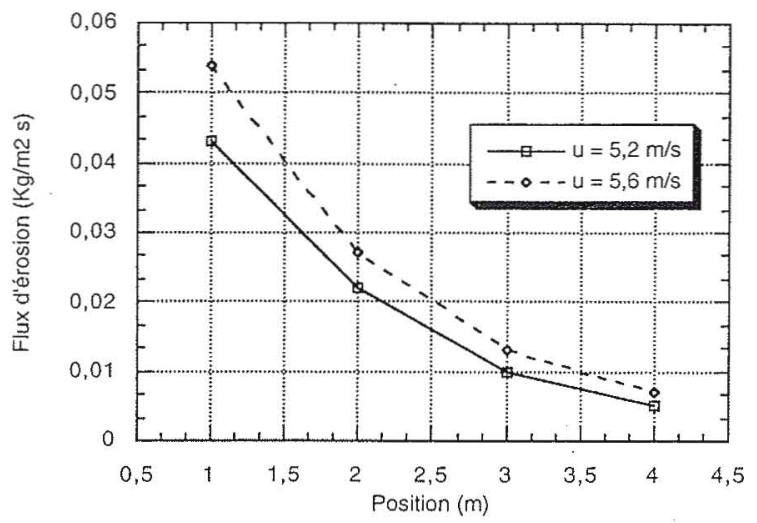

6 - Variation du flux de masse érodée entre l'amont et l'aval.

4. 2 Effet de la vitesse d'érosion et de la masse volumique

Si on compare les dérivées temporelles de l'épaisseur du lit des particules pour les matériaux $\mathrm{A}$ et $\mathrm{C}$, on se rend compte de l'effet de la vitesse-seuil de frottement. Le tableau suivant présente ces dérivées.

On observe clairement le rôle que joue la vitesse-seuil de frottement. Ce rôle consiste à limiter l'arrachement des particules. Malgré cet effet, et à cause de la forte densité du matériau $C\left(1410 \mathrm{~kg} / \mathrm{m}^{3}\right)$, le flux massique de ce dernier est plus fort que celui du matériau A $\left(544 \mathrm{~kg} / \mathrm{m}^{3}\right)$.

Ces mesures vont nous permettre de fixer les conditions aux limites au niveau du sol pour la modélisation numérique développée [4], [5]. Pour cela, il ne suffit pas seulement de connaitre le flux d'érosion, mais il est nécessaire également de connaître la proportion de masse qui est effectivement transférée par la couche de saltation à la couche de suspension (fig 7).

\section{FLUX DE SALTATION ET FLUX DE DIFFUSION}

Les mesures de la masse érodée nous permettent d'estimer le flux massique de la couche de saltation. En effet, et grâce aux mesures des profils de concentration dans la couche de suspension [4], on peut déterminer le flux de masse dans cette couche. Le flux de saltation est la différence entre le flux d'érosion et le flux de suspension.

\begin{tabular}{|c|c|c|}
\hline Type de particules & Dérivée temporelle & Flux massique \\
\hline $\mathrm{A}: \mathrm{u}_{\mathrm{w}_{\mathrm{t}}}=0,134 \mathrm{~m} / \mathrm{s}$ & $9,910^{-5} \mathrm{~m} / \mathrm{s}$ & $0,054\left(\mathrm{Kg} / \mathrm{m}^{2} \mathrm{~s}\right)$ \\
\hline $\mathrm{C}: \mathrm{u}_{\mathrm{s}_{\mathrm{t}}}=0,143 \mathrm{~m} / \mathrm{s}$ & $4,810^{-5} \mathrm{~m} / \mathrm{s}$ & $0,068\left(\mathrm{Kg} / \mathrm{m}^{2} \mathrm{~s}\right)$ \\
\hline
\end{tabular}

Tableau 2. - Effet de la vitesse-seuil de frottement. 
1

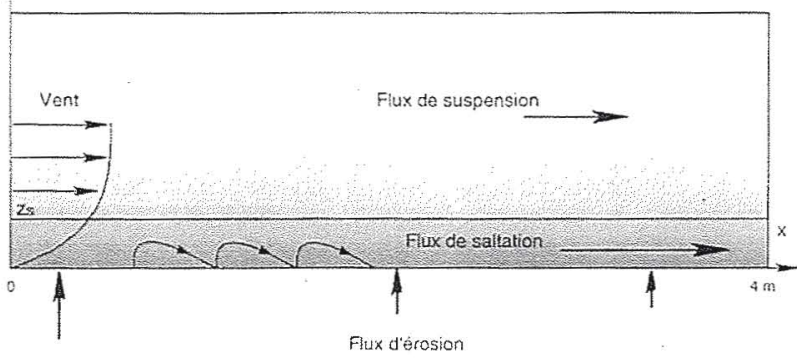

7 - Schéma représentant les trois flux.

Le flux massique dans la couche de suspension est donné par la formule suivante:

$\Phi_{\text {suspension }}=\int_{z=z_{\text {saltation }}}^{\infty} u(z) c(z) d z=\int_{0,02 \mathrm{~m}}^{0,0467 \mathrm{~m}} \mathrm{j}(\mathrm{z}) c(z) d z=\sum_{i=1}^{50} u_{i} c_{i}=0,00545 \frac{\mathrm{kg}}{\mathrm{m}^{2} \mathrm{~s}}$

où $c_{i}$ est la concentration mesurée à la hauteur $z_{i}$ et $u_{i}$ est la vitesse définie par :

$u_{i}=\frac{u_{*}}{0,41} \log \left(\frac{z_{i}}{z_{0}}\right)$

(avec $z_{0}=0,0001 \mathrm{~m}$ et $u_{*}=0,6 \mathrm{~m} / \mathrm{s}$ )

Le flux massique produit par l'érosion est donné par l'intégrale suivante :

$$
\Phi_{\text {érosion }}=\int_{x=0}^{4 m} \phi_{\text {érosion }}(x) d x
$$

où $\phi_{\text {crosion }}$ est représenté par l'ajustement suivant à partir des mesures:

$$
\phi_{\text {érosion }}=(0,036809-0,02458 \log x)
$$

Ainsi l'équation (5) devient

$\Phi_{\text {érosion }}=\int_{x=0,1 \mathrm{~m}}^{4,1 \mathrm{~m}}(0,03681-0,02458 \log \mathrm{x}) \mathrm{dx}=0,12560 \frac{\mathrm{kg}}{\mathrm{m}^{2} \mathrm{~s}}$

Le flux massique dans la couche de saltation s'obtient grâce à la conservation de la masse par la différence entre le flux massique érodé par l'écoulement et le flux massique dans la couche de suspension :

$$
\Phi_{\text {saltation }}=\Phi_{\text {érosion }}-\Phi_{\text {suspension }}=0,12015 \frac{\mathrm{kg}}{\mathrm{m}^{2} \mathrm{~s}}
$$

Le rapport a entre les flux de suspension et d'érosion donne:

$$
\alpha=\frac{\Phi_{\text {suspension }}}{\Phi_{\text {érosion }}}=\frac{0,00545 \frac{\mathrm{kg}}{\mathrm{m}^{2} \mathrm{~s}}}{0,12560 \frac{\mathrm{kg}}{\mathrm{m}^{2} \mathrm{~s}}}=0,04339
$$

c'est-à-dire que seulement $4 \%$ de la masse des particules prélevées sont mis en suspension.

\section{VI}

Nous avons obtenu des mesures quantitatives du flux de masse érodée par un écoulement turbulent sur un lit de particules disposé dans une soufflerie diphasique. Cette modélisation physique permet de définir les conditions limites au niveau du sol, qui sont utilisées pour une modélisation numérique du transport de neige en suspension.

A partir de ces mesures, nous avons analysé :

- l'apparition des ondulations à la surface du lit de particules : en effet, à cause du phénomène de reptation, des ripples apparaissent et se déplacent dans le sens de l'écoulement, engendrant une évolution ondulatoire de l'épaisseur du lit.

- le flux massique d'érosion : ce flux est déterminé ainsi que son évolution en fonction de la position et de la vitesse de l'écoulement. Le flux croît avec la vitesse de l'écoulement et décroît linéairement entre l'amont et l'aval de la veine d'étude.

- la proportion saltation/diffusion : la détermination des profils de concentration dans la couche de suspension permet de déterminer le flux de masse en suspension. Le flux d'érosion diminué du flux de suspension donne le flux massique dans la couche de saltation. On peut ainsi quantifier la masse transportée dans cette couche : elle représente plus de 90\% de la masse totale prélevée dans le cas de nos essais.

\section{Bibliographie}

[1] Greeley, R., and J.D. Iversen, Wind as a Geological Process on Earth, Mars, Venus and Titan, Cambridge University Press, Cambridge, 333, 1985.

[2] Clos-Arceduc, A., Essai d'explication des formes dunaires sahariennes. Etudes de photo. Interprétation $N^{\circ} 4$, Revue de l'Institut Géographique National, 66 p., 1969.

[3] Anderson, R.S., A theoretical model for aeolian impact ripples, Sedimentology, 34, 943-956, 1987.

[4] Martinez, H., Contribution à la modélisation du transport éolien de particules. Mesures de profils de concentration en soufflerie diphasique. Thèse de l'Université Joseph Fourier, 204 p., 1996.

[5] Naaim-Bouvet, F., M. Naaim et H. Martinez, Profils de concentration de la neige soufflée : théorie, résolution numérique et validation expérimentale in situ, La Houille Blanche, Revue Internationale de l'Eau) n5-1996. 